\title{
Combined Fluoroscopic and Nerve Stimulator Technique for InJection Of the Piriformis MUSCLe
}

\author{
Andres Betts, MD \\ The diagnosis of radicular pain second- \\ ary to Piriformis Muscle Syndrome has been \\ historically difficult due to lack of accurate \\ means to identify and selectively inject the \\ piriformis muscle. This paper describes a \\ simple and reproducible technique to safely \\ inject the piriformis muscle, using a combina- \\ tion of fluoroscopic guidance and a standard \\ neuro-muscular stimulator. \\ Key words: piriformis muscle syndrome, \\ fluoroscopy, nerve stimulator
}

A less common but important cause of lower extremity radicular pain may result from nerve irritation by the piriformis muscle, known as Piriformis Muscle Syndrome. This syndrome must be differentiated from other causes of sciatic type pain, more typically related to lumbar disc disease or spinal stenosis, and may go largely under-diagnosed. The piriformis muscle syndrome was first described in 1947 by Robinson (1), who ascribed several clinical features: a history of trauma to gluteal region, pain in the sciatic notch extending down the lower extremity, pain worsened by lifting or bending over but improved with traction, a palpable and tender piriformis muscle (positive Lasegue sign), and gluteal atrophy. In addition, patients may manifest pain on forced external rotation of the hip (positive Freiberg's sign). This constellation of findings has been amended to also include negative imaging studies to rule out lumbar disc disease, nerve impingement, or other causes of radicular pain. As a result, the syndrome is often a diagnosis of exclusion (2).

The piriformis muscle originates from S2-S4 along the ventrolateral margin of the sacrum and extends through the greater sciatic notch, ultimately inserting onto the piriformis fossa of the greater trochanter. The muscle functions to externally rotate the thigh while the hip is in flexion. The sciatic nerve (formed from

Andres Betts, MD, San Clemente Community Hospital, Pain Service, 654 Camino delos Mares, San Clemente, CA 92673

E-mail: abettsmd@cox.net

Support:There was no external funding in preparation of this manuscript

Conflict of Interest: None
L4-S3 nerve roots) also passes through the sciatic notch and is typically located anterior to the piriformis muscle. However, six possible variations on the anatomic lie of the nerve relative to the piriformis muscle have been described in cadaveric studies (3).

The proper diagnosis and treatment of Piriformis Muscle Syndrome has been historically difficult, due to lack of techniques to accurately and reproducibly inject the piriformis muscle with local anesthetic- with or without steroid preparation. Two techniques have been previously described- one involving the use of a nerve stimulator and surface landmarks, while the other utilizes combined fluoroscopic and electromyographic guidance, with injection of contrast dye to obtain a confirmatory myogram $(4,5)$. With successful identification of the piriformis muscle the diagnosis is more assured and patients may better respond to standard injected medication. The non-fluoroscopic technique utilizes standard surface landmarks similar to those for performing a sciatic block, combined with the use of a standard nerve stimulator to better define the location of the piriformis muscle. The piriformis is identified by a reduction of frank gluteal contraction as the insulated needle traverses through the gluteal muscle and encounters the deeper-lying piriformis muscle. The piriformis produces a small detectable twitch of the needle and slight abduction at the hip. There are anomalous anatomic arrangements, however, where the sciatic nerve is dorsal to the piriformis muscle and may be encountered prior to the piriformis muscle. This would prohibit deeper passage of the needle to the more ventrally located piriformis, for fear of direct damage to the sciatic nerve. These anatomic arrangements may limit the wide application of this non-fluoroscopic technique.

The other technique, which applies electromyography combined with fluoroscopic guidance, is a highly accurate method; however, it does require expertise in the use electromyographic techniques and the availability of the necessary equipment. These two factors may limit the wide use of this method.

Knowledge on the use of a standard nerve stimulator is generally widespread among pain specialists, and the device is simple and readily available in most facilities. In this paper I describe a successful technique that utilized fluoroscopic guidance along with the use of a nerve stimulator, as an alternative method to reliably define the piriformis muscle.

\section{Details of Procedure}

After obtaining full informed consent the patient with suspected piriformis muscle syndrome is placed prone on a suitable fluoroscopy table. The buttock area on the affected side is widely prepped and draped in a sterile fashion. AP view of the right hemi-pelvis and acetabular region is obtained. The most inferior aspect of the SI joint and of the most superior-lateral aspect of the right acetabulum is identified, then a metal marker is placed on a line adjoining these two structures, about $1 / 3$ of the way medial from acetabular landmark. Local infiltration with $1 \%$ Lidocaine may be used for local anesthetic of the skin but is avoided in deeper structures to prevent loss of stimulating abili- 
Table 1. Piriformis Muscle Stimulation Parameters for 8 patients

\begin{tabular}{|c|c|}
\hline Initial Output (mA) & Minimal Output Achieved (mA) \\
\hline 1.5 & 4.0 \\
\hline 1.2 & 0.2 \\
\hline 1.5 & 0.4 \\
\hline 2.0 & 0.8 \\
\hline 2.0 & 0.4 \\
\hline 1.0 & 0.3 \\
\hline 1.0 & 0.6 \\
\hline 3.8 & 0.8 \\
\hline
\end{tabular}

The stimulator needle was inserted into the substance of the piriformis muscle, using fluoroscopic guidance. In some patients, the needle was advanced until bony contact was made with the dorsal surface of the ileum. The needle was then pulled back less than $1 \mathrm{~cm}$ and adjusted until a twitch could be re-established at $\leq 0.8 \mathrm{~mA}$ output. Generally, the twitch slowly decreased until only the hub of the needle was seen to move, or until minimal movement of the gluteal region at the lowest possible stimulator output was noted. In each case the twitch was abolished with injection of $3 \mathrm{ml}$ of $0.25 \%$ bupivacaine. All patients reported between $70-100 \%$ pain reduction following injection of local anesthetic.

ty of the nerve stimulator by the local anesthetic.

Following satisfactory superficial local anesthetic, a 4-inch or longer 22-gauge Stimuplex insulated needle (B Braun Medical, Bethlehem, PA) is inserted and advanced with fluoroscopic guidance and nerve stimulation output at 1.5-2.0 milliamps and $2 \mathrm{hz}$ frequency. Initially contraction of the gluteus maximus muscle is typically obtained and the stimulator

output is reduced untill only a moderate gluteal twitch is observed. The needle should then be additionally advanced until contraction of the gluteus is markedly diminished. The output may then be reduced to less than $0.6 \mathrm{~mA}$ and the needle tip adjusted until there is just a discernable twitch noted at the needle hub and at the hip. Occasionally the dorsal aspect of the Ileum is encountered and the needle should be pulled back 1-2 mm. The out-

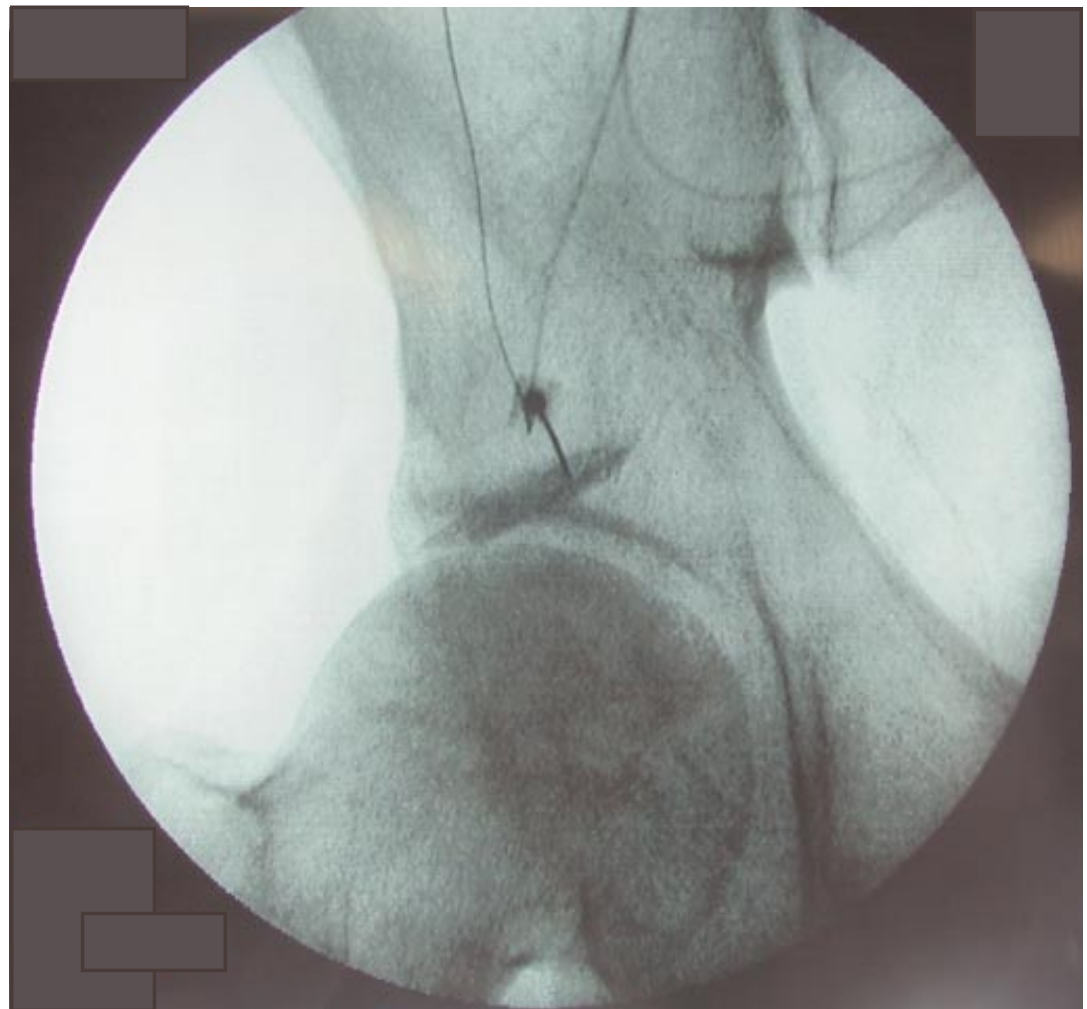

Fig 1. Myogram of Piriformis Muscle with Combined Fluoroscopic and Nerve Stimulator Localization. put is then adjusted until a slight twitch is obtained, which identifies the piriformis muscle. There must be no paresthesias or contractions of the calf or foot muscles, indicating too close proximity to the sciatic nerve. Typical stimulation data are shown in Table 1 . One $\mathrm{ml}$ of radiographic contrast material may be injected to obtain a satisfactory myogram (Fig 1). A syringe should be prepared with $3 \mathrm{ml}$ total volume of $0.25 \%$ bupivacaine with or without steroid medication and injected into the piriformis muscle after negative aspiration for blood. The patient may often experience discomfort with the actual injection of solution, but is usually only transient and resolves as the local anesthetic takes effect.

Following the procedure patients should note relief of their usual pain. Occasionally, patients may experience some slight difficulty rotating the body about the hip while standing on the involved leg, or some slight weakness with external rotation at the right hip.

Most patients have responded well to a single or perhaps only one repeat injection. In patients that are refractory to local and/or steroid medication, BOTOX injection has been utilized as an alternative therapy $(6,7)$. One patient, in this series of more than 12 , had recurrence of her pain and was successfully treated with BOTOX 100 units in 2 $1 / 2 \mathrm{cc}$ total volume of $0.25 \%$ Bupivacaine into the piriformis muscle, using the same above described technique.

\section{DISCUSSION}

The diagnosis of Piriformis Muscle Syndrome is one that is difficult to make on clinical grounds alone and often difficult to treat. This report describes the application of a novel, yet simple injection technique to successfully identify the piriformis muscle.

Visualization of the muscle with fluoroscopy aids in needle placement, and electrical twitch corroborates that the needle is within the piriformis muscle tissue. Accurate placement of the needle with this approach may reduce the risk of complications possible with this injection, such as unintentional injection of the sciatic nerve, and improve the diagnostic accuracy of the technique.

Due to variations in physician training and patient anatomy, this simple alternative approach may be of value to the 
pain practitioner. The role of the piriformis muscle in radicular pain may be more effectively addressed with accurate diagnosis and precision injection of medication.

Author Affiliation:
Andres Betts, M.D.
San Clemente Community Hospital
Pain Service
654 Camino delos Mares
San Clemente, CA 92673
E-mail: abettsmd@cox.net

\section{RefERENCES}

1. Robinson D. Piriformis syndrome in relation to sciatic pain. Am / Surg 1947;73: 355-358.

2. Prizale JR, Hudkins TH, Fishman LM. The Piriformis Syndrome. Am J Orthop 1996;819-823.

3. Simons D, Travell J. Myofascial Origins of Low Back Pain. Postgrad Med 1993; 73: 99-108.

4 Fishman SM, Caneris OA, Bandman TB, Audette JF, Borsook D. Injection of the Piriformis Muscle by Fluoroscopic and
Electromyographic Guidance. Reg Anesthesia Pain Med 1998;23:554-559.

5. Hanania, M. New Technique for Piriformis Muscle Injection using a nerve Stimulator. Reg Anesth 1997; 22:200-202.

6. Fishman LM, Anderson C, Rosner B. Botulinum toxin type $A$ and Physical Therapy in the Treatment of Piriformis Syndrome. Am J Phys Med Rehabil 2002;81: 936-42.

7. Childers MK, Wilson DJ et al. Botulinum Toxin Type A Use in Piriformis Muscle Syndrome: A Pilot Study. Am J Phys Med Rehabil 2002;81: 751-759. 
